\title{
Comparative Study of OFDM and CDMA Technique
}

\author{
Lalit Singh GariA ${ }^{1}$, Amit Shah $^{2}$, Deepesh Rawat ${ }^{3}$ \\ ${ }^{1}$ (Asst. Prof.(Ece), Btkit,Dwarahat Uttarakhand, India) \\ ${ }^{2}\left(\right.$ Mtech $\left(D c 2^{\text {nd }}\right.$ Year) Btkit,Dwarahat, Uttarakhand, India) \\ ${ }^{3}\left(\right.$ Mtech (Dc $2^{\text {nd }}$ Year) Btkit,Dwarahat, Uttarakhand, India)
}

\begin{abstract}
Orthogonal frequency division multiplex (OFDM) modulation is being used more and more in telecommunication both in wired and wireless. This modulation technique has several advantages, reason for its increasing usage in communication. OFDM can provide high data rates, it can be implemented easily, it is spectrally efficient and with sufficient robustness to channel imperfections. On the other hand most third generation mobile phone systems are using Code Division Multiple Access (CDMA) as their modulation technique. For this reason, CDMA is also investigating so that the performance of both CDMA and OFDM can be compared. It is found that OFDM performs extremely well when compared with CDMA, and provide very high tolerance to multipath delay spread, channel noise, and peak power clipping. In addition to this it provides a high spectral efficiency The noise performance of OFDM is found to depend solely on the modulation technique used for modulating each carrier of the signal. The OFDM signal performance is found to be the same as for a single carrier system, using the same modulation technique and can provide large data rates with sufficient robustness to radio channel impairments The minimum signal to noise ratio (SNR) required for BPSK was $\sim 7 d B$, where as it was $\sim 12 d B$ for QPSK and $\sim 25 d B$ for 16PSK. CDMA was found to perform poorly in a single cellular system, with each cell only allowing 7-16 simultaneous users in a cell, compared with 128 for OFDM.1.25 MHz bandwidth and 19.5 kbps user data rate was used for it. This low cell capacity of CDMA was attributed to the use of non-orthogonal codes used in the reverse transmission link, leading to a high level of inter-user interference.
\end{abstract}

Keywords: BER, CDMA, FFT, ISI, OFDM, OFDM, S/N

\section{INTRODUCTION}

The telecommunications' industry is in the midst of a veritable explosion in Wireless technologies Wireless communication is one of the most active areas of technology development. Wireless Broadband Technologies allow the simultaneous delivery of voice, data and video over fixed or mobile platforms. Wi-Fi, WiMAX, LTE, UMB are some of the emerging technologies. With the increase demand there is a need to transmit information wirelessly, quickly, and accurately. To address this need, several technologies have been combined for high rate transmission with forward error correction techniques.Frequency division multiplexing (FDM) is a technology that transmits multiple signals simultaneously over a wired or a wireless system in which each signal is limited by a specific frequency band, and is modulated by data stream. Orthogonal Frequency Division Multiplexing[1] and is a special case of frequency division multiplexing, where the data is distributed over a large number of carriers, which are orthogonal to each other. OFDM[2] is spectrally efficient compared to the conventional FDM systems, since it doesn't need guard bands between adjacent channels. This orthogonality property of the carriers is main characteristic of OFDM, since the interference due to the other carriers is prevented when the receiver demodulates a particular carrier. Once the OFDM data are modulated to time signal, all carriers transmit in parallel to fully occupy the available frequency bandwidth [3].OFDM is becoming widely applied in wireless communications systems due to its high rate transmission capability with high bandwidth efficiency and its robustness with regard to multi-path fading and delay [4]. One of the principal advantages of OFDM is its utility for transmission at very nearly optimum performance in unequalized channels and in multipath channels. Inter-symbol interference (ISI)[5] and inter-carrier interference (ICI) can be entirely eliminated by the simple expedient of inserting between symbols a small time interval known as guard interval. One of the principal disadvantages of OFDM is sensitivity to frequency orset in the channel. There are two deleterious effects caused by frequency orset; one is the reduction of signal amplitude in the output of the filters matched to each of the carriers and the second is introduction of ICI from the other carriers which are now no longer orthogonal to the filters. Timing errors also affects OFDM system performance by reducing the delay spread robustness and by introducing phase shift in the received spectrum. Adaptive estimation of channel is necessary before the demodulation of OFDM signals since the wireless channel is frequency selective and time varying. Some of the main advantages of OFDM are efficient spectral usage and its multi-path delay spread tolerance by allowing overlapping in the frequency domain. Another significant advantage is that the modulation and demodulation can be done using IFFT and FFT operations, which are computationally efficient. 


\section{OFDM MODEL USED IN SIMULATIONS}

An OFDM system is modelled using Matlab[6] to allow various parameters of the system to be varied and tested. The aim of doing the simulations is to measure the performance of OFDM system under different channel conditions, and to allow for different OFDM configurations to be tested. An OFDM system model used is shown in Figure 1. As it can be seen in the figure channel imperfections are modelled in this implementation. In the end of the transmission, when the receiver receives the data, a comparison of the transmitted and the received messages is done in order to calculate the Bit Error Ratio (BER). This paper does not explain in detail the simulation code. It uses it to create results and see the behavior of OFDM under different channel properties.

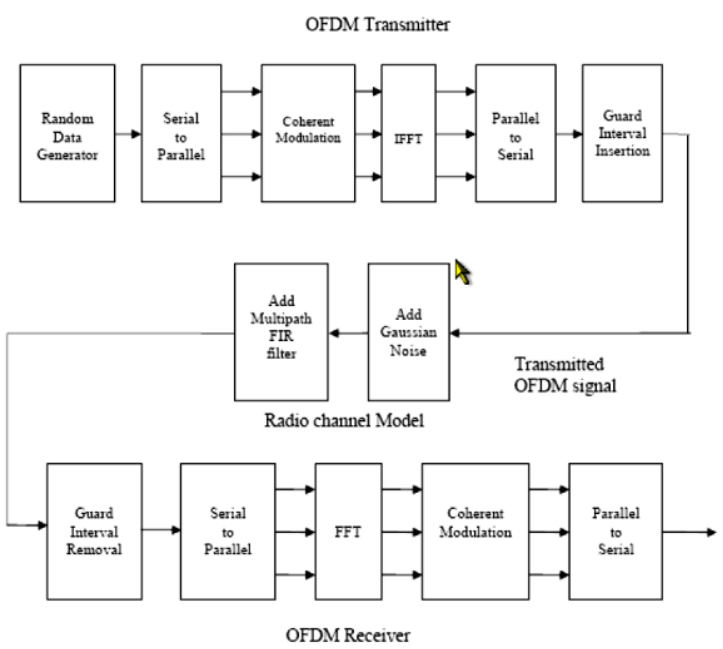

Fig 1: OFDM Simulation Flowchart

\section{CODE Division MulTiPl ACCESS}

In communication network systems multiplexing is a term used to refer to a process where multiple analog message signals or digital data streams are combined into one signal over a shared medium. The multiplexed signal is transmitted over a link, which may be wire or wireless . In a multiuser system, capacity is certainly a sensitive issue. The efficiency with which the available resources are used determines the number of users the system can support. Higher efficiency is achieved if the resources are made available to all users and they are assigned according to demand. This characterizes what is known as demand-assigned multiple-access (DAMA) or, simply, multiple access.

Code division multiple access (CDMA) is a multiple access technique where different users share the same physical medium and same frequency band simultaneously. The main ingredient of CDMA is the spread spectrum technique, which uses high rate signature pulses to enhance the signal bandwidth far beyond what is necessary for a given data rate. In CDMA different users can be identified and separated at the receiver by means of their characteristic individual signature pulses (sometimes called the signature waveforms) by their individual codes. Nowadays, the most prominent applications of CDMA are mobile communication systems like CDMA One (IS-95), UMTS or CDMA 2000. To apply CDMA in a mobile radio environment, specific additional methods are required to be implemented in all these systems. Methods such as soft handover and power control have to be applied to control the interference by other users and to be able to separate the users by their respective codes. Spread spectrum means enhancing the signal bandwidth far beyond what is necessary for a given data rate and thereby reducing the power spectral density (PSD) of the useful signal so that it may even sink below the noise level. This is a desirable property for military communications because it helps to hide the signal and it makes the signal more robust against intended interference (jamming). Spreading is achieved by a multiplication of the data symbols with spreading sequence of pseudo random signals. These sequences are called pseudo noise (PN) sequences or code signals.

\subsection{Capacity of OFDM and CDMA with Multiple Cells}

With any cellular system, interference from neighbouring cells lowers the overall capacity of each cell. For conventional TDMA and FDMA systems, each cell must have a different operating frequency from its immediate neighbouring cells. This is to reduce the amount of interference to an acceptable level. The frequencies are reused in a pattern, with the spacing between cells using the same frequency which is determined by the reuse factor. The required frequency reuse factor depends on the interference tolerance of the transmission system. Analog systems typically require a carrier to interference ratio $(\mathrm{C} / \mathrm{I})$ of greater then $18 \mathrm{~dB}$ [7], which requires reuse factor of $1 / 7$ (see Figure 2 (b)). Most digital systems only require a C/I of $12 \mathrm{~dB}$, 
allowing a reuse factor of 1/3-1/4 (see Figure 2 (a)). CDMA uses the same frequency in all cells, and hence ideally allowing a reuse factor of 1 (see Figure 2).
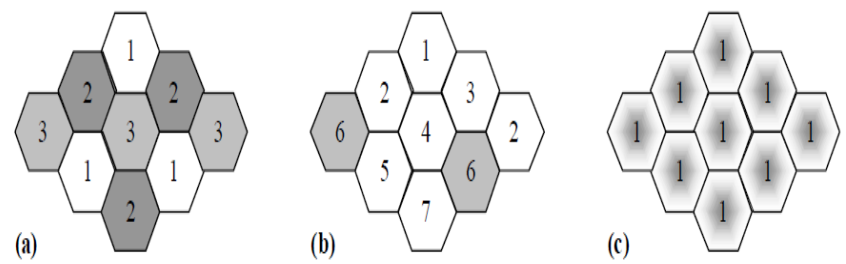

Fig 2: Frequency reuse patterns for (a) 3 frequencies (Digital systems), (b) 7 frequencies (Analog FDMA), (c) CDMA In practice the frequency reuse efficiency of CDMA is low, as neighbouring cells cause interference, which reduce user capacity of both. The frequency reuse factor for a CDMA system is about 0.65 [8]. Figure 3 shows the interference from neighbouring cells. Most of the neighbouring interference is from the immediate neighbours of the cell.

A.

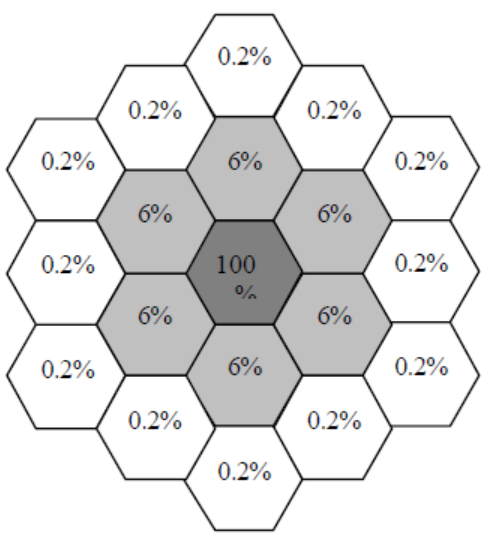

Fig 3: Interference contributions from neighbouring cells in a CDMA system.

The cell capacity for a multi-cellular CDMA system equal to the single cell capacity reduced by the frequency reuse factor. The cell capacity for CDMA is very low if cell sectorization and voice activity detection is used. A straight CDMA system can only have somewhere between $5-11$ users/cell/1.25MHz. Using voice activity detection and cell sectorization allow the capacity to be increased by up to 6.4 time, allowing somewhere between 30-70 user/cell/1.25MHz. OFDM require a frequency reuse pattern to be used in a multi-cellular environment to reduce the level of inter-cellular interference. The $\mathrm{C} / \mathrm{I}$ required must be greater then $\sim 12 \mathrm{~dB}$. This could be done with a frequency reuse factor of $\sim 3$. This should be easily achived as cell sectorization could also be used to reduce the level of interference. This would result in the cell capacity for an OFDM system of approximately $128 / 3=42.7$ users/cell/1.25MHz in a multicellular environment

\subsection{Forward Link}

\section{SIMULATION MODEL-:}

The forward link of the CDMA system modelled uses orthogonal Walsh codes to separate the users. Walsh code is randomly allocated to each usera to spread the data to be transmitted. The transmitted signals from all the users are combined together and then passed through a radio channel model. This allows for adding multipath interference, clipping of the signal and adding white gaussian noise to the signal.The receiver uses the same Walsh code that was used by the transmitter to demodulate the signal and recover the data. Using the Walsh code received signal has been despread and then it is sub-sampled back down to the original data rate. This is done by using an integrate and dump filter which is followed by a comparator to decide whether the data was a 1 or a 0 . The original data is then compared with received data to been demodulated and filtered is compared with the expected amplitude of the signal based on the transmitted data. The RMS amplitude error directly relates to the bit error rate, and is a useful measurement to make. 


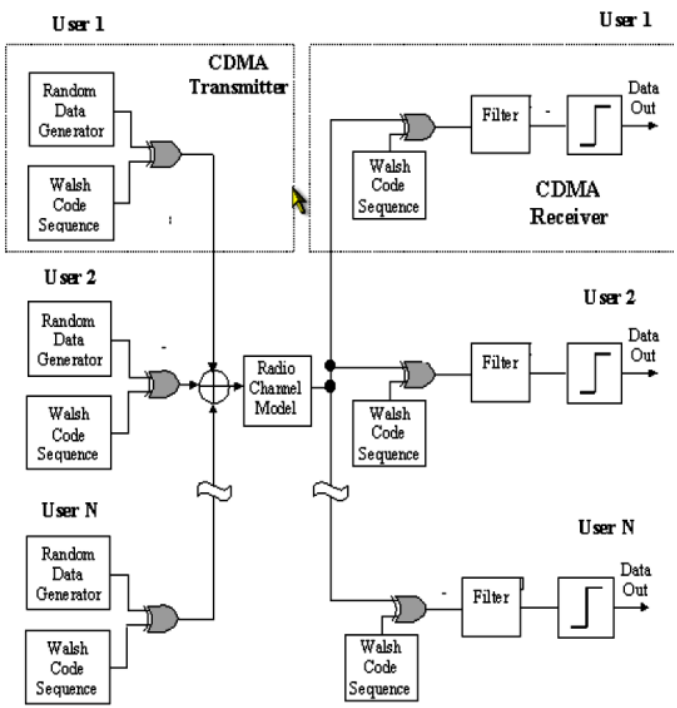

Figure 3 Model used for the CDMA forward link.

\subsection{Reverse Path}

In the reverse link of the CDMA, orthogonal Walsh code are not used as in the case of forward link and rest of the simulation is similar in both reverse link and forward. As shown earlier it is extremely difficult to use orthogonal codes effectively in a reverse link from the mobiles to the base station, because of the difficulty in synchronizing the system accurately enough. Because of this simply long pseudo random codes were used instead of the Walsh codes

V. COMPARISON BETWEEN BPSK AND QPSK BASED OFDM

\section{Simulation Results for OFDM with BPSK:}

FFT size $=64$;

Number of Sub-carries $=64$;

Bits transmitted $=10^{\wedge} 5$;

Cyclic Prefix length $=16$;

Channel parameters $=\left[\begin{array}{lll}.23 & .96 & .23\end{array}\right]$

FFT size $=64$;

Number of Sub-carries=64;

Bits transmitted $=10^{\wedge} 5$;

Cyclic Prefix length=16;

Channel parameters $=\left[\begin{array}{lll}.23 & .96 & .23\end{array}\right]$

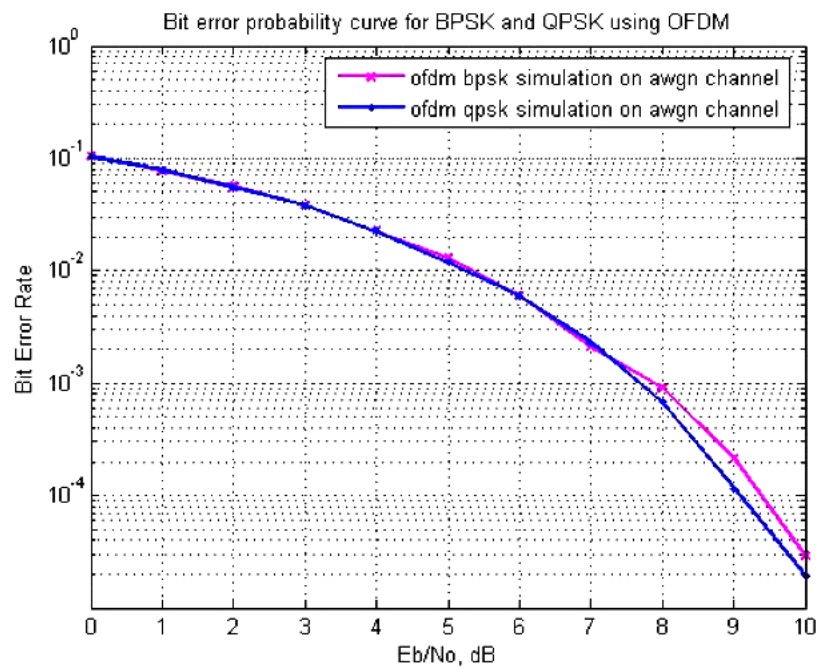

Simulation Results for CDMA: 


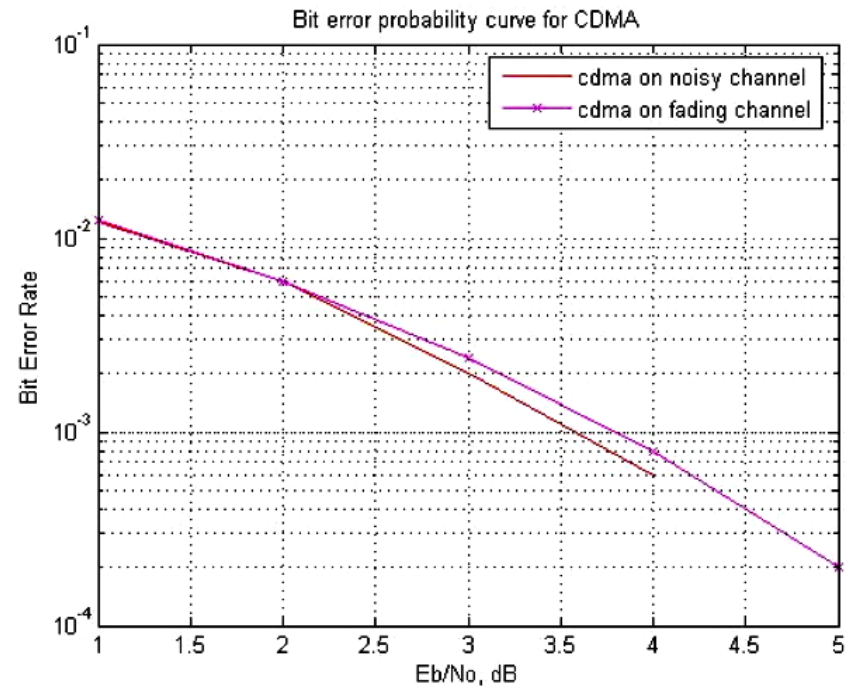

VI. CONCLUSION

In this paper, a revision of basic concepts regarding orthogonal frequency division multiplex (OFDM) has been carried out. As compared with CDMA OFDM was found to perform very well, with it out performing CDMA in many areas for a single and multicell environment. OFDM was found to allow up to 2-10 times more users than CDMA in a single cell environment and from 0.7 - 4 times more users in a multi-cellular environment. The difference in user capacity CDMA and OFDM was dependent on whether cell sectorization and voice activity detection is used. It is found that CDMA only performs well in a multi-cellular environment where a single frequency is used in all cells. This increases the comparative performance against other systems that require a cellular pattern of frequencies to reduce inter-cellular interference. One major area, which hasn't been investigated, is the problems that may be encountered when OFDM is used in a multiuser environment. One possible problem is that the receiver may require a very large dynamic range in order to handle the large signal strength variation between users .

\section{Future Work}

With the increasing requirements for future wireless applications, CDMA may be considered as the most important for wireless systems. This system has the ability to incorporate very large bandwidth without sacrificing equalization complexity. In this paper though we have studied about the principles of CDMA and OFDM we haven't implemented the channel estimation techniques as in the case of OFDM. But the same can be carried out using pilot and implicit training sequence techniques to optimize the equalizer performance. Also lies the feasibility study of Multiple Input Multiple Output (MIMO) OFDM systems. In this study we have discussed about about Single Input Single Output (SISO) OFDM system. MIMO OFDM can be implemented using multiple transmitting and receiving antennas which is an interesting future work

\section{References}

[1] Y. Mostofi and D. C. Cox, "Mathematical analysis of the impact of timing synchronization errors on the performance of an OFDM system," IEEE Trans. Commun., vol. 54, no. 2, pp. 226-230, Feb. 2006

[2] A Brief History of OFDM http://www.wimax.com /commentary/wimax_weekly/sidebar-1-1-a-brief-history-of-ofdm

[3] Heiskala, Juha and Terry, John. OFDM Wireless LANs: A Theoretical and Practical Guide SAMS 2001]

[4] P. H.Moose, "A technique for orthogonal frequency division multiplexing frequency offset correction," IEEE Trans. Commun., vol. 42, no. 10, pp. 2908-2914, Oct. 1994.

[5] H. Steendam and M. Moeneclaey, "Analysis and optimization of the performance of OFDM on frequency-selective time-selective fading channels," IEEE Trans. Commun., vol. 47, no. 12, pp. 1811-1819, Dec. 1999.

[6] Orlandos Grigoriadis "Ber Calculation Using Matlab Simulation For Ofdm Transmission”, IMECS 2008, 19-21 March, 2008, Hong Kong

[7]. J. D. Gibson, "The mobile communications handbook", CRC Press, pp. 366-368, 1996

[8]. D. Whipple, "North American Cellular CDMA", Hewlett-Parkard Journal, pp. 90-97, December 1993.

[9] Edfors, O., Sandell, M., Van de Beek, J.-J., Landström, D., and Sjöberg, F., An Introduction to Orthogonal Frequency Division Multiplexing, Luleå, Sweden: Luleå Tekniska Universitet, 1996, pp. 1-58. 